\title{
A Rare Case of Metastases to Paranasal Sinus From Colonic Adenocarcinoma
}

\author{
Aaditya Prakash ${ }^{1}$, Amitabh Kumar Upadhyay ${ }^{2}$ \\ 1. Radiation Oncology, Tata Main Hospital, Jamshedpur, IND 2. Medical Oncology, Tata Main Hospital, Jamshedpur, \\ IND \\ Corresponding author: Aaditya Prakash, aadityaprakash07143@gmail.com
}

\begin{abstract}
Metastases of malignant tumors to the nasal cavity and paranasal sinuses are very rare. Metastases to these locations are usually solitary and produce similar symptoms to those of a primary sinonasal tumor. Pain, nasal obstruction, and epistaxis are the most common symptoms. Although any malignancy could potentially lead to metastasis to the paranasal sinuses, colo-rectal malignancy metastasizes to this site is rare.

We report a case of metastatic adenocarcinoma of colorectal origin to the paranasal sinuses in a 55-year-old female who was initially diagnosed with adenocarcinoma of the colon with lung and liver metastasis. She subsequently developed metastasis to left ethmoidal and sphenoidal sinuses during treatment. A histologic study of the surgical specimen from the sinonasal cavity demonstrated a tumor identical to the patient's prior primary tumor of the colon. The sinonasal neoplastic tissue showed marked positivity for carcinoembryonic antigen and expressed cytokeratin 20, which differentiates metastatic colonic adenocarcinoma from primary intestinal-type adenocarcinoma (ITAC). She received palliative radiation therapy but died three months after the diagnosis.
\end{abstract}

These subsets of patients have a poor prognosis. In the majority of patients, palliative therapy is the only possible treatment option. Nevertheless, whenever possible, surgical excision either alone or combined with radiotherapy may be useful for palliation of symptoms and, rarely, to achieve prolonged survival.

Categories: Pain Management, Radiation Oncology, Oncology

Keywords: metastatic colo-rectal cancer, palliative radiation therapy, rare metastases, sino-nasal metastasis

\section{Introduction}

The overall incidence of primary adenocarcinoma of the nasal cavity and paranasal sinuses probably accounts for $10 \%$ to $20 \%$ of all sinonasal malignancies [1]. Most of these tumors are of salivary gland origin. Some tumors are of rare patterns and have histology similar to those of adenocarcinoma of the colon. Metastases of malignant tumors to the nasal cavity and paranasal sinuses are very rare. Incidences of these metastases are common in the 50 and 60 years age group of men and 60 and 70 years age group of women [2]. A review of literature on tumors of paranasal sinuses reports very few cases of sinonasal metastases from Review ended 04/26/2021 Published 04/27/2021

\section{(c) Copyright 2021}

Prakash et al. This is an open access article distributed under the terms of the Creative Commons Attribution License CC-BY 4.0., which permits unrestricted use, distribution, and reproduction in any medium, provided the original author and source are credited.

\section{colorectal carcinoma [3-8].}

\section{Case Presentation}

Our patient is a 55-year-old female who presented initially to the general surgery clinic in March 2016 after noticing altered bowel habits for a duration of two months. Her past medical history was associated with type 2 diabetes mellitus and hypertension. She had no previous surgical history, no prior colonoscopies, and no family history of colon cancer. Her only gastrointestinal complaint was the changes in the bowel pattern. There was no history of any melena, hematochezia, or weight loss. A colonoscopy demonstrated a splenic flexure mass obstructing the transverse colon lumen and biopsies of the lesion revealed moderately differentiated adenocarcinoma (Figure 1). 


\section{Cureus}

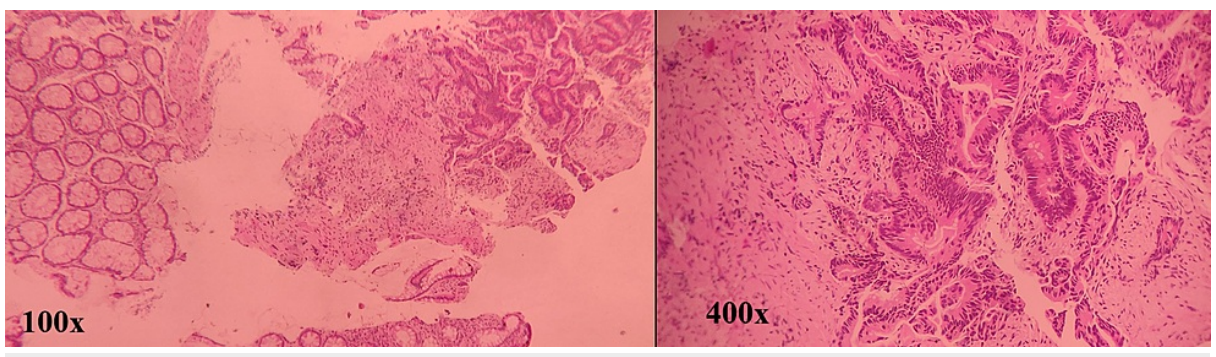

FIGURE 1: H\&E stained colonic endoscopic biopsy sections showing infiltrative, branching glands lined by atypical cells: $100 x$ and $400 x$

H\&E: hematoxylin and eosin.

Serum carcino-embryonic antigen (CEA) was markedly elevated at $88.52 \mathrm{ng} / \mathrm{mL}$. Computed tomography (CT) scans of the thorax, abdomen, and pelvis were obtained, showing a mass in the transverse colon and pericolonic adenopathy with evidence of lung and liver metastatic disease. She received four cycles of systemic chemotherapy with the FOLFOX4 regime. Interval CT scans were suggestive of complete regression of lung metastasis. The residual lesion was seen in liver segments of VI and VII. She underwent an extended right hemicolectomy with end colostomy in September 2016 followed by radiofrequency ablation (RFA) of liver metastatic lesions in October 2016. She completed a further course of FOLFOX4 till November 2016. She was kept on routine follow-up as per National Comprehensive Cancer Network (NCCN) guidelines.

She underwent a whole-body PET-CT scan as part of a follow-up in June 2017. Findings were suggestive of isolated fluorodeoxyglucose (FDG) avid para-aortic lymph nodes. She underwent exploratory laparotomy with retroperitoneal lymph node dissection (RPLND) and side to side ileo-sigmoid anastomosis in July 2017 followed by 12 cycles of chemotherapy with FOLFIRI regime till May 2018. Her follow-up CT scans of the thorax, abdomen, and pelvis showed an increase in the number and size of pulmonary nodules with an increase in the number of para-aortic lymph nodes. Biopsy from retroperitoneal lymphadenopathy was suggestive of metastatic adenocarcinoma with K-RAS and N-RAS-wild type. She was started on CAPEOX with Bevacizumab-based chemotherapy. In between chemotherapy treatment schedules, she was presented with pain in the left eye with diminution of vision for 15 days. There was an associated left-side facial headache. On eye examination, there was a vision of 6/6 in the right eye and no vision in the left eye. There was a divergent squint in the left eye, at the time of presentation. Contrast-enhanced MRI of the brain and paranasal region revealed mucosal thickening of size $3.2 \times 2.6 \mathrm{~cm}^{2}$ in the left ethmoidal sinus and sphenoid sinus with extension along the medial wall of the left orbit. Functional endoscopic sinus surgery (FESS) was done. Histopathology reported it as neoplastic etiology (Figure 2).

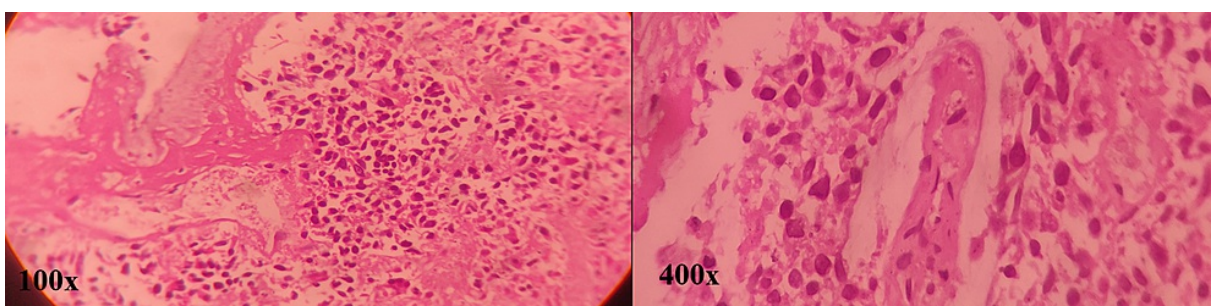

FIGURE 2: H\&E stained section of a fragmented sphenoidal bone biopsy showing scattered metastatic clusters of atypical epithelial cells: $100 \times$ and $400 x$

H\&E: hematoxylin and eosin.

Immunohistochemistry reported as adenocarcinoma of intestinal type with the positivity of cytokeratin (CK) 20, CDX2, CEA, and negative for TTF1, CK7 immunoprofile. The report was consistent with a colon as primary.

Due to an increase in severity of pain in the left eye and facial region, the patient was planned for palliative radiotherapy to the local site with Intensity Modulated Radiotherapy Technique (IMRT). She received a planning target volume (PTV) dose of $30 \mathrm{~Gy}$ in 10 fractions on Varian TrueBeam ${ }^{\mathrm{TM}}$ with IMRT (Varian Medical Systems, Inc., Palo Alto, CA) (Figure 3). She tolerated it very well and got significant pain relief at the local site. 


\section{Cureus}

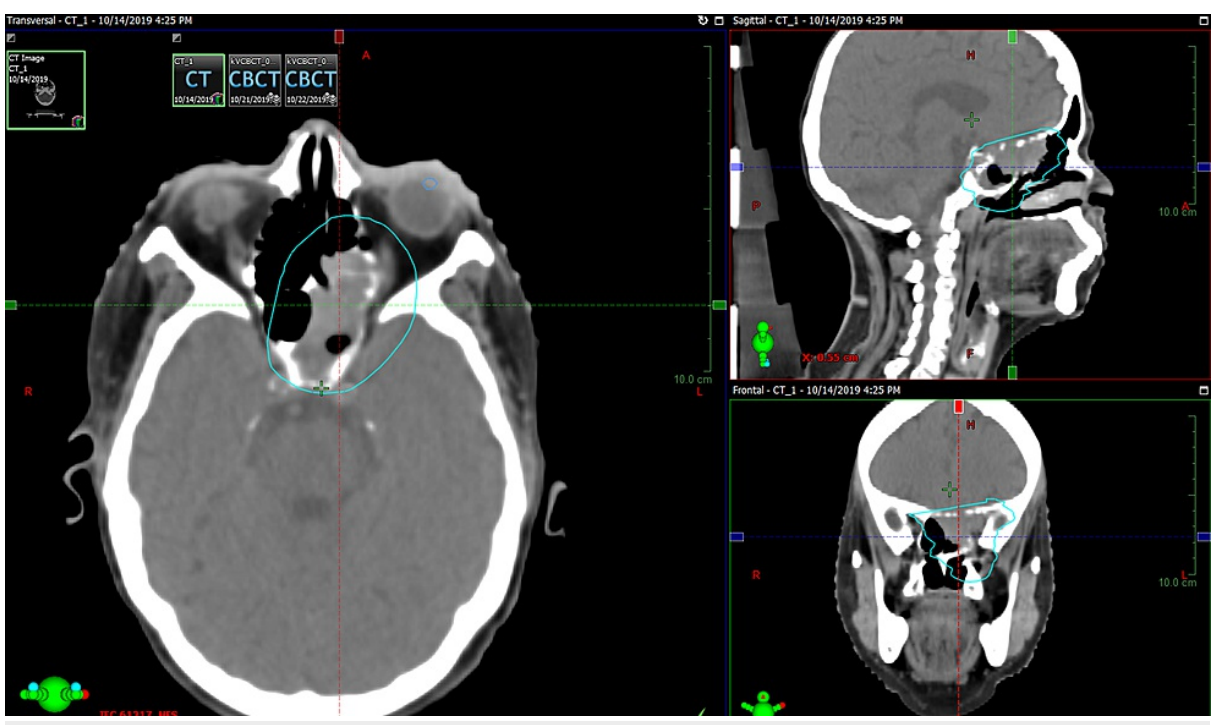

FIGURE 3: Planning target volume coverage in planning CT scan in all sections (axial, sagittal, coronal; clockwise)

She continued on palliative chemotherapy but succumbed subsequently after three months of palliative radiotherapy due to progressive disease and complications.

\section{Discussion}

The most common presentation of symptoms in primary and metastatic malignancy of nasal cavity and paranasal sinuses are facial pain, recurrent epistaxis, and nasal obstruction $[9,10]$. It is important to distinguish between primary and metastatic malignancies. Most challenging is to differentiate between histology of colonic metastases to these sites from primary sinus adenocarcinomas, more specifically the colonic variant of intestinal-type adenocarcinoma (ITAC) [11].

Primary ITAC is a rare primary malignancy of the nasal cavity and paranasal sinuses. Generally linked to occupation-related hazard, especially with wood dust exposure, but sometimes occurs sporadically [12]. As the histology of the ITAC-colonic variant is difficult to differentiate from metastatic colorectal cancer, therefore, a diagnosis of metastatic adenocarcinoma from these sites requires correlation with any prior history of colorectal cancer. Immunohistochemical studies of the biopsy specimen play an important role to formulate the diagnosis. Positivity for CDX2, CK20, and negativity for CK7 differentiates metastatic colonic adenocarcinoma from ITAC $[11,13]$. The CK20+/CK7- immunoprofile is considered to be specific for colorectal epithelial tumors [14].

In 1940, Batson postulated that through the low-pressure valveless system connecting deep pelvic veins, intercostal veins, vena cava, and the azygous system, retrograde metastasis to nasal and paranasal sinuses occurs, during increased intrathoracic and abdominal pressure [15].

Nasal and paranasal metastases from colorectal cancer are associated with poor outcomes. None of the cases reports five-year survival from the diagnosis of these metastases. All cases were treated with palliative radiotherapy to the nasal and paranasal region $[10,11,16]$. The mean survival post-palliative radiotherapy was between 2 and 18 months after diagnosis of the metastases [11,16].

\section{Conclusions}

In conclusion, paranasal metastasis is a rare condition and it is important to be differentiated from primary ITAC. The presence of paranasal metastases, unlike ITAC, represents advanced disease and carries a grave prognosis. These types of patients should be managed symptomatically with palliative radiation therapy or chemotherapy with the best supportive care.

\section{Additional Information}

\section{Disclosures}

Human subjects: Consent was obtained or waived by all participants in this study. Conflicts of interest: In compliance with the ICMJE uniform disclosure form, all authors declare the following: Payment/services info: All authors have declared that no financial support was received from any organization for the 
submitted work. Financial relationships: All authors have declared that they have no financial relationships at present or within the previous three years with any organizations that might have an interest in the submitted work. Other relationships: All authors have declared that there are no other relationships or activities that could appear to have influenced the submitted work.

\section{References}

1. Weber AL, Stanton AC: Malignant tumors of the paranasal sinuses: radiologic, clinical, and histopathologic evaluation of 200 cases. Head Neck Surg. 1984, 6:761-76. 10.1002/hed.2890060310

2. Izquierdo J, Armengot M, Cors R, Pérez A, Basterra J: Hepatocarcinoma: metastases to the nose and paranasal sinuses. Otolaryngol Head Neck Surg. 2000, 122:932-3. 10.1016/s0194-5998(00)70032-5

3. Sera K, Yajin H, Tagashira S: A case of metastatic maxillary cancer from sigmoid colon cancer . Pract Oct. 1988, 27:89-96

4. Robinson D: Antral metastases from carcinoma. J Laryngol Otol. 1973, 87:603-9. 10.1017/s0022215100077379

5. Shadyev KD, Dal̆niak AA, Zmolro AS: [Metastasis of cancer of the stomach to the maxillary sinus] . Vestn Otorinolaringol. 1989, 85-6.

6. Friedmann I, Osborn DA: Metastatic tumours in the ear, nose and throat region. J Laryngol Otol. 1965 79:576-91. 10.1017/s0022215100064100

7. Kent SE, Majumdar B: Metastatic tumours in the maxillary sinus. A report of two cases and a review of the literature. J Laryngol Otol. 1985, 99:459-62. 10.1017/s0022215100097048

8. Reino AJ: Factors in the pathogenesis of tumors of the sphenoid and maxillary sinuses: a comparative study . Laryngoscope. 2000, 110:1-38. 10.1097/00005537-200010001-00001

9. Azarpira N, Ashraf MJ, Khademi B, Asadi N: Distant metastases to nasal cavities and paranasal sinuses case series. Indian J Otolaryngol Head Neck Surg. 2011, 63:349-52. 10.1007/s12070-011-0269-8

10. López F, Devaney KO, Hanna EY, Rinaldo A, Ferlito A: Metastases to nasal cavity and paranasal sinuses . Head Neck. 2016, 38:1847-54. 10.1002/hed.24502

11. bin Sabir Husin Athar PP, bte Ahmad Norhan N, bin Saim L, bin Md Rose I, bte Ramli R: Metastasis to the sinonasal tract from sigmoid colon adenocarcinoma. Ann Acad Med Singapore. 2008, 37:788-90.

12. Barnes L: Intestinal-type adenocarcinoma of the nasal cavity and paranasal sinuses . Am J Surg Pathol. 1986, 10:192-20. 10.1097/00000478-198603000-00006

13. Bien S, Kaminski B, Okla S, Kopczynski J: Metastasis of rectal adenocarcinoma to the skull base and paranasal sinuses, with unusual clinical symptoms. Otolaryngol Pol. 2005, 59:627-30.

14. Kende AI, Carr NJ, Sobin LH: Expression of cytokeratins 7 and 20 in carcinomas of the gastrointestinal tract . Histopathology. 2003, 42:137-40. 10.1046/i.1365-2559.2003.01545.x

15. Batson OV: The function of the vertebral veins and their role in the spread of metastases . Ann Surg. 1940, 112:138-49. 10.1097/00000658-194007000-00016

16. Kaminski B, Kobiorska-Nowak J, Bien S: Distant metastases to nasal cavities and paranasal sinuses, from the organs outside the head and neck. Otolaryngol Pol. 2008, 62:422-5. 10.1016/S0030-6657(08)70284-1 